\title{
GUIDE-Modell: Ein Kompass für den Beratungstisch
}

\author{
Christine Andres Roduit • Marie-Christine Walter
}

Eingegangen: 22. Februar 2021 / Angenommen: 16. März 2021 / Online publiziert: 1. Juni 2021

(C) The Author(s) 2021

Zusammenfassung Karrierecoaches und Laufbahnberatenden sowie Klient/innen ein Modell zur Verfügung zu stellen, das eine selbstgesteuerte und erfolgreiche Laufbahngestaltung stützt - diesem Ziel folgte die öffentliche Berufs-, Studienund Laufbahnberatung Oberwallis bei der Entwicklung des Guide-Modells. Dieses basiert auf prozessorientierten Laufbahnberatungsmodellen und inhaltsorientierten Ressourcenmodellen. Anhand eines Fallbeispiels aus dem Karrierecoaching wird die Anwendung des GUIDE-Modells in der Praxis veranschaulicht. Erkenntnisse aus der Praxisarbeit mit dem Modell, welches als Drehscheibe auf dem Beratungstisch vorliegt, werden dargelegt und diskutiert.

\section{Schlüsselwörter Karriere-Ressourcen · Prozessberatung · Visualisierung}

\section{GUIDE model: A compass for the consultation desk}

Abstract Providing a model for career coaches and counsellors as well as for clients that follows self-directed and successful career construction-that's the goal of the Educational and Vocational Guidance Office of the Upper Valais in developing the GUIDE model. It is based on process-oriented career advice models and contentoriented resource models. The use of the GUIDE model is demonstrated in practice through a case study from career coaching. Lessons learned from working with the model, which is presented as a rotating disc on the consultation desk, are set out and discussed.

Keywords Career resources · Process consultation · Visualization

Christine Andres Roduit $(\bowtie) \cdot$ Marie-Christine Walter $(\bowtie)$

Amt für Berufs-, Studien- und Laufbahnberatung Oberwallis, Schlossstraße 30, 3900 Brig-Glis,

Schweiz

E-Mail: christine.andres-roduit@admin.vs.ch; marie-christine.walter@admin.vs.ch 


\section{Einleitung}

Der Titel GUIDE und die Kompassform des Beratungsmodells, das in diesem Beitrag vorgestellt wird, beziehen sich auf den inneren Kompass, der dem Menschen Orientierung bietet, wenn durch die heutige ungewisse Zeit vertraute Anker gelöst werden. Der innere Kompass symbolisiert die Orientierung an persönlichen Werten und die Selbstgestaltung des eigenen Weges. Auch mit Mobilität, Offenheit für Veränderungen und Lernbereitschaft kann er in Verbindung gebracht werden.

Für die Beratungspersonen der öffentlichen Berufs-, Studien- und Laufbahnberatung Oberwallis (BSL Oberwallis) waren und sind die Entwicklung des GUIDEModells, dessen Implementierung in ein umfassendes Beratungskonzept (BSL Oberwallis 2018, S. 38 ff.) sowie die Umsetzung von Konzept und Modell in die Praxis mit einem jahrelangen (Selbst)reflexions-, Lern- und Entwicklungsprozess verbunden. Die dadurch gewonnene, theoretisch fundierte und praxisnahe Grundlage bietet den Beratungspersonen Orientierung und vereinfacht die gegenseitige Verständigung in einer gemeinsamen Beratungshaltung. Das GUIDE-Modell ist prozessorientiert und inhaltlich flexibel. Es kann zur Beratung von diversen Zielgruppen im Kontext unterschiedlicher Anliegen eingesetzt sowie unter Einsatz von traditionellen und modernen Methoden und Arbeitsmitteln angewendet werden. In der BSL Oberwallis dient das GUIDE-Modell als Referenzrahmen im Karrierecoaching ${ }^{1}$, in der Laufbahnberatung, in der Studienwahl und in der ersten Berufswahl.

In diesem Beitrag werden das GUIDE-Modell sowie dessen Entwicklung und Einsatz in der Coaching-Praxis präsentiert. Es wird gezeigt, wie das Modell als Drehscheibe auf dem Beratungstisch zur Visualisierung genutzt wird. Aus der Perspektive der Beratungsperson werden Erkenntnisse aus der Arbeit mit dem GUIDEModell dargelegt, wobei der Frage nachgegangen wird, wie Prozess- und Expertenberatung sinnvoll integriert werden können. Zunächst werden einige Begriffe, die für die Themen und Zielsetzungen dieses Beitrags wesentlich sind, definiert und voneinander abgegrenzt.

\section{Begriffliche Klärung}

Coaching ist eine Form der Beratung, die sich an psychisch gesunde Menschen richtet (Greif 2008, S. 59). Nach Rauen (2014, S. 2 f.) handelt es sich beim Coaching um einen interaktiven und personenzentrierten Begleitungsprozess. Coaching ist zeitlich begrenzt, lösungsorientiert und zielfokussierend. Gegenseitige Akzeptanz und Vertrauen sowie Freiwilligkeit charakterisieren die Coaching-Arbeit. Beim Coaching geht es gemäß Rauen um Hilfe zur Selbsthilfe, und daher beabsichtigt Coaching immer eine Verbesserung der Selbstreflexions- und Selbstmanagementfähigkeiten der Klient/innen. Loebbert (2018, S. 22f.) versteht Coaching als Prozessberatung, welche das Ziel verfolgt, die Selbststeuerung von Personen zu verbessern. Eine Verbesserung der Selbststeuerung beim Handeln geht nach Loebbert mit einer passenden Veränderung der bisherigen Denk-, Fühl- und Handlungsmuster einher.

\footnotetext{
${ }^{1}$ Die Begriffe „Karriere“ und „Laufbahn“ werden in diesem Bericht synonym verwendet.
} 
Karrierecoaching ist ein Coachingformat, das darauf ausgerichtet ist, Klient/innen „bei Laufbahnfragen und Anliegen des Laufbahnmanagements zu unterstützen“ (Ebner und Kauffeld 2019, S. 761). Im Karrierecoaching sollen Klient/innen die Lösung für ein persönliches Thema selbst herleiten und das notwendige Zutrauen für die erfolgreiche Umsetzung der Lösung im Coachingprozess aufbauen (Kauffeld et al. 2018, S. 292).

Berufs- und Laufbahnberatung als eine spezifische Anwendung der Beratungspsychologie (Hirschi 2019, S. 740) richtet sich an psychisch gesunde Personen und betrachtet das Individuum nicht losgelöst, sondern immer im Bezug zum jeweiligen Kontext. Hirschi (2018, S. 16ff., 2019, S. 740f.) postuliert ein modernes Verständnis von Berufs-, Studien- und Laufbahnberatung, indem Klient/innen insbesondere darin unterstützt werden,

- eine subjektiv bedeutungsvolle Identität zu konstruieren, ihre Selbstreflexion zu steigern und eine ihrer persönlichen Identität und Lebensgeschichte entsprechende Laufbahn zu gestalten,

- persönliche und soziale Ressourcen anzuwenden und zu entwickeln, Laufbahngestaltungskompetenzen zu fördern und ihre Laufbahn aktiv zu gestalten,

- ihr Privat- und ihr Arbeitsleben in Einklang zu bringen, mit unvorhergesehenen Ereignissen in der Laufbahn und mit Belastungen umzugehen,

- Informationen auszuwählen, zu bewerten und anzuwenden.

Der Einsatz von Testverfahren und Expertenwissen spielen eine Rolle, sie stehen jedoch nicht im Zentrum einer modernen Berufs- und Laufbahnberatung. Stattdessen treten vielmehr Aspekte der Selbstreflexion und Selbstkonstruktion in den Vordergrund (Hirschi 2019, S. 741). Schreiber (2020, S. 30f.) weist darauf hin, dass der Fokus in einer nachhaltig wirksamen Berufs-, Studien- und Laufbahnberatung auf die Prozessberatung zu legen ist, wobei Beratende insbesondere Wissen und Können zur Prozess- und Veränderungsbegleitung sowie Stärkung des Zutrauens erwerben sollten. Moderne Ansätze der Berufs- und Laufbahnberatung bilden die Grundlage des GUIDE-Modells, wie es im Folgenden dargelegt wird.

Unterschiede zwischen Laufbahnberatung und Karrierecoaching: Der in der Literatur wohl meist genannte und wichtigste Unterschied besteht darin, dass es im Coaching um Prozessberatung geht, bei der keine Wissensvermittlung verfolgt wird. In einer Laufbahnberatung hingegen wird im Prozess konkretes Wissen vermittelt. Gemäß Ebner und Kauffeld (2019, S. 763) ist es diese inhaltliche Enthaltsamkeit, mit der Karrierecoaching sich deutlich von der Laufbahnberatung abgrenzt.

Unterschiede zwischen Prozessberatung und Expertenberatung: Nach Schein (2010, S. 38) zielt Prozessberatung darauf ab, ,die Lernfähigkeit des Klientensystems zu erhöhen, damit es zukünftige Probleme selbst lösen kann“. Bei der Expertenberatung hingegen ,wird das Problem behoben“. Expertenberatung ist gemäß Schein (2010, S. 25) dann sinnvoll, wenn Klient/innen Informationen und eine Dienstleistung benötigen, die sie selber nicht erbringen können. Die Beratungsperson soll stets beurteilen, welche Rolle für die jeweilige Situation angemessen und für den Aufbau einer helfenden Beziehung förderlich ist (Schein 2010, S. 41). Loebbert (2018, S. 10f.) zeigt anhand des Modells des Kontinuums auf, dass die Unterscheidung zwischen Prozessberatung und Expertenberatung nicht strikt, son- 
dern kontinuierlich ist. Es geht nach Loebbert um die Frage, wie viel Prozessund wie viel Expertenberatung für den Handlungserfolg der Klient/innen respektive deren Anliegen nötig sind.

\section{Wissenschaftlicher und praktischer Stand der Karriereberatung sowie Verortung des GUIDE-Modells}

Fortschritte in Technologien, Veränderungen in Organisationsstrukturen, neue Arbeitsformen und veränderte Einstellungen gegenüber der Arbeit haben in den letzten 40 Jahren den Kontext beruflicher Laufbahnen stark verändert. Auch die Verantwortung für die Karriereentwicklung hat sich zusehends von den Organisationen auf die Arbeitnehmenden verschoben (Hirschi 2015, S. 65 f.). Diese Veränderungen gehen mit zunehmender Wahlfreiheit, jedoch auch mit Unsicherheit und mehr Eigenverantwortung der Arbeitnehmenden einher. Der psychologische Vertrag beinhaltet gemäß Lang-von Wins und Triebel (2012, S. 19ff.) implizite Annahmen und Erwartungen zwischen Arbeitnehmende/r und Organisation, welche nicht formell festgehalten sind und sich dynamisch verändern. Nach einer modernen Auffassung des psychologischen Vertrags leisten Mitarbeitende durch ihren Einsatz für Aufgaben und Projekte einen Beitrag zum Unternehmenserfolg und erhalten als Gegenleistung vom Unternehmen die Möglichkeit, sich eigenverantwortlich weiterzuentwickeln und somit beschäftigungsfähig zu bleiben. Mit traditionellen Laufbahntheorien, wie z. B. der Person-Umwelt-Passung, können Anforderungen und Probleme, die sich im aktuellen beruflichen Kontext ergeben, nicht mehr ausreichend erklärt und bewältigt werden (Masdonati et al. 2019, S. 12).

Was ist notwendig, um die eigene Laufbahn erfolgreich zu gestalten? Seit Jahrzehnten beschäftigt sich die Forschung mit dieser Frage. Seit den 1970er-Jahren erklären Autoren (z.B. Schein 1978) das Entstehen von Karrieren verstärkt durch Wechselwirkungen zwischen Organisationen und Arbeitnehmenden (Latzke et al. 2019, S. 7). Schein (1975) erarbeitete das Konzept der Karriere-Anker, das in der Folge mehrfach ergänzt und weiterentwickelt wurde (vgl. Schein 1978, 2004; vgl. Rappe-Giesecke 2008). Schein unterscheidet zwischen einer inneren (subjektiven) und einer äußeren (objektiven) Karriere. Seit Anfang der 1990er-Jahre sind in der Laufbahnforschung zahlreiche, eher psychologisch orientierte Ansätze und Konzepte entstanden (Latzke et al. 2019, S. 7), die sich teilweise überschneiden (Hirschi 2012, S. 372).

Verschiedenste Aspekte sind gemäß Hirschi (2015, S. 68 ff.) wichtig, um die eigene Laufbahn in der heutigen dynamischen Arbeitswelt erfolgreich zu gestalten, wie z. B. das Konzept der Laufbahn-Adaptabilität, der Beschäftigungsfähigkeit sowie proteische Karriere-Orientierungen. Unter einer proteischen Karriere-Orientierung (Hall 2004, S. 3 ff.) wird ein wertegeleitetes und selbstbestimmtes Handeln in Bezug auf die eigene Laufbahnentwicklung verstanden. Sie beinhaltet die aktive Anpassung an sich immer wieder ändernde Arbeitsbedingungen. Eine solche auf den subjektiven Erfolg ausgerichtete Einstellung erleichtert das Erreichen von Zufriedenheit und eine bessere Integration von beruflichen und außerberuflichen Lebensbereichen (Hirschi 2015, S. 72). Diesen modernen Konzepten ist gemeinsam, dass sie ihren Fokus auf 
die individuelle Autonomie bei Laufbahnentscheiden richten und die zunehmende Relevanz von subjektivem statt objektivem Laufbahnerfolg betonen (Gubler 2019, S. 957). In der Praxis des Personalmanagements und des Laufbahncoachings könnte das Potenzial dieser neuen Laufbahnmodelle (z.B. proteische Karriere-Orientierung) noch vermehrt genutzt werden (ebd., S. 956 f.).

Mit dem Ziel, diverse Forschungsbefunde und Modelle zur erfolgreichen Laufbahnentwicklung und Berufswahl übersichtlich zusammenzufassen, hat Hirschi (2012) das Karriere-Ressourcen-Modell entwickelt (vgl. Hirschi 2015, S. 73). Die Weiterentwicklung dieses Modells (Hirschi et al. 2018) erlaubt die Erfassung von 13 Karriere-Ressourcen (Schlüsselfaktoren) für Karriereerfolg.

Verortung des GUIDE-Modells: Zur selbstgesteuerten und erfolgreichen Laufbahngestaltung integriert das GUIDE-Modell die Karriere-Ressourcen sowie proaktive Karrieremanagement-Verhaltensweisen (Hirschi 2012; Hirschi et al. 2018) in vier Prozessphasen und gewährleistet die konkrete Umsetzung in die Praxis, indem es zu jeder Phase Methoden und Arbeitsinstrumente vorschlägt. Es spiegelt wichtige Aspekte einer zeitgemäßen Laufbahnberatung wider. Karriere-Ressourcen, die für die Arbeitsmarktfähigkeit zentral sind, werden in der Beratung nach dem GUIDEModell erfasst, zielgerichtet genutzt und weiterentwickelt. Je nach Anliegen der Klient/innen ermöglicht das GUIDE-Modell entsprechend auch die Ausrichtung des inneren Kompasses, und somit lassen sich Verbindungen zu anderen Konzepten und Modellen auf der Praxisebene finden, welche die Klient/innen auf der Suche nach ihrer Berufung, einem erfüllten Leben (vgl. Gulder 2013) oder in der Weiterentwicklung ihrer beruflichen Identität (vgl. Scheidt 2009) unterstützen. Die Beratungsarbeit nach dem GUIDE-Modell lässt sich folglich mit verschiedenen weiteren Ansätzen aus Forschung und Praxis kombinieren. Das triadische Denken z. B., das in der Triadischen Karriere-Beratung (Rappe-Giesecke 2008) zur Anwendung kommt, kann in der Laufbahnberatung nach dem GUIDE-Modell neue Blickwinkel eröffnen. Es lässt sich gut mit dem Karriere-Ressourcen-Management verbinden.

\section{Entwicklung des GUIDE-Modells in der BSL Oberwallis}

Anlass für die Entwicklung des GUIDE-Modells war der Wunsch nach einer theoriegeleiteten Struktur, anhand welcher Klient/innen ganzheitlich, effizient und wirksam in ihrer selbstgesteuerten Laufbahnentwicklung unterstützt werden können. Nach einer Explorationsphase wurde der Fokus auf wissenschaftlich fundierte und theoriegestützte Modelle zu Karriere-Ressourcen und dem proaktiven Laufbahnmanagement (Hirschi 2012; Hirschi et al. 2018) gerichtet. Diese Modelle beschreiben, welche Ressourcen bei Erwachsenen für den subjektiven (z. B. Sinnhaftigkeit der Arbeit) und den objektiven Laufbahnerfolg (z. B. hohes Einkommen) wichtig sind. Auch für den Erhalt und die Förderung von Arbeitsmarktfähigkeit sind Karriere-Ressourcen von Bedeutung. Bei Jugendlichen verkörpern Karriere-Ressourcen Indikatoren von Berufswahlbereitschaft (Haenggli et al. 2019, S. 7).

Um Karriere-Ressourcen in der Beratungsarbeit zielgerichtet zu nutzen und weiterzuentwickeln, benötigt es deren Einbettung in definierte Prozessphasen. Vor diesem Hintergrund wurde in der BSL Oberwallis das GUIDE-Modell entwickelt. Es 
basiert sowohl auf prozessorientierten Laufbahnberatungsmodellen als auch auf inhaltsorientierten Ressourcenmodellen (Hirschi 2011, 2012, 2020; Sampson et al. 1999; Hirschi et al. 2018). Eine Beratung nach dem GUIDE-Modell soll Klient/ innen darin unterstützen, ihre Laufbahn langfristig selbst zu gestalten und ihre Arbeitsmarktfähigkeit zu erhalten und zu fördern.

Das GUIDE-Modell wurde in einer ersten Version in der Fachzeitschrift Panorama publiziert (Andres Roduit et al. 2018). Inzwischen wurde das GUIDE-Modell auf nationaler Ebene aufgegriffen. Im Rahmen des Pilotprojekts ,viamia - kostenlose Standortbestimmung für Erwachsene ab 40 Jahren" "2 strukturiert es den Beratungsprozess.

\section{Das GUIDE-Modell}

Die Buchstaben G-U-I-D-E bilden die Anfangsbuchstaben der Beratungsmodule ab: Gap, Understanding and Improving, Developing solutions, Execution (BSL Oberwallis 2018, S. 39f.). Der Titel des Modells GUIDE nimmt Bezug auf den ,,path with a heart" (Hall 2004, S. 9) und den ,inneren Kompass“ (Hirschi 2011, S. 308) sowie auf den ,inneren Wegweiser, den die Menschen zum Gestalten ihrer beruflichen Laufbahn benötigen“ (BSL Oberwallis 2018, S. 39). Diese Perspektive ist in der grafischen Darstellung des GUIDE-Modells durch die Kompassform versinnbildlicht (s. Abb. 1).

Im Zentrum des GUIDE-Modells stehen die Aktivitäten (proaktive Laufbahnmanagement-Verhaltensweisen) und die Karriere-Ressourcen (Hirschi 2012; Hirschi et al. 2018). Die Ressourcen, welche sich gegenseitig beeinflussen (vgl. Hirschi 2012, S. 377 f.), sind sinnbildlich als farbige, ineinandergreifende Zahnräder dargestellt. Durch die Aktivitäten werden die Zahnräder angetrieben respektive die Ressourcen genutzt und weiterentwickelt. Die Zahnräder treiben sich auch gegenseitig an, was bedeutet, dass Ressourcen andere Ressourcen stärken können. Ist ein Zahnrad beeinträchtigt, behindert es andere Zahnräder. So kann auch ein Mangel in einer Ressource die Entwicklung anderer Ressourcen verhindern. Im GUIDE-Modell sind die Karriere-Ressourcen und Aktivitäten zu den vier Prozessphasen ausgerichtet, in welchen sie zielgerichtet bearbeitet werden (gelbe Bereiche in Abb. 1). Diese vier Beratungsmodule sind ineinandergreifend und spiralförmig dargestellt, da sie sich in der Praxis nicht immer klar voneinander abgrenzen lassen.

\subsection{Inhalte des GUIDE-Modells}

Das GUIDE-Modell fokussiert nachfolgende Karriere-Ressourcen und Aktivitäten (Hirschi 2012, S. 374 ff.; Hirschi et al. 2019).

Der Bereich Wissen und Kompetenzen beinhaltet neben Bildung, Erfahrung, beruflicher Expertise und fachübergreifenden Kompetenzen auch Informationsressourcen (z.B. Kenntnisse über Bildungssystem, Arbeitsmarkt). Umfeld-Ressourcen sind außerhalb der Person angesiedelt. Es handelt sich um Entwicklungsmöglichkeiten

\footnotetext{
2 www.berufsbildung2030.ch/de/21-projekte-de/52-standortbestimmung, Zugegriffen: 15.01.2021.
} 


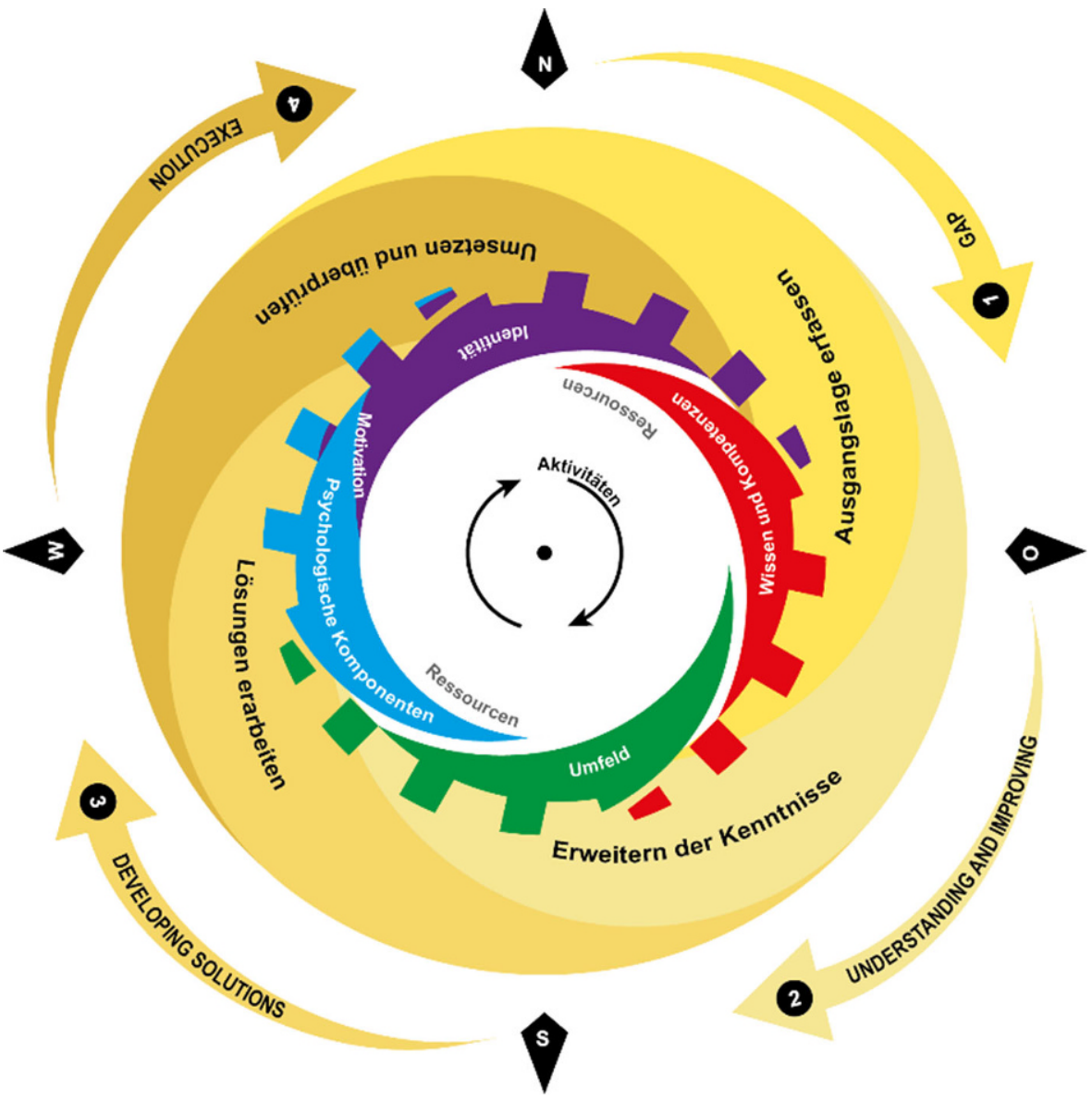

Abb. 1 GUIDE-Modell ( BSL Oberwallis 2018, 2020)

im derzeitigen Unternehmen, Arbeitsherausforderung in der aktuellen Arbeitsstelle, Unterstützung durch Institutionen oder Freunde und Familie in der beruflichen Entwicklung usw. Einen weiteren Ressourcenbereich stellt die Identität dar: Inwieweit nimmt Arbeit eine zentrale Rolle im Leben der Person ein, und inwieweit ist sich die Person über persönliche Interessen, Werte, Rollen, Ziele und Zielkongruenz (Lebenspläne) im Klaren. Psychologische Komponenten beinhalten sowohl stabilere förderliche Persönlichkeitsmerkmale (z.B. Gewissenhaftigkeit) als auch leichter veränderbare Merkmale, Kognitionen und Einstellungen wie z. B. Zutrauen in die eigene Fähigkeit, die berufliche Laufbahn selbstständig erfolgreich zu gestalten. Auch Hoffnung, Karriere-Optimismus (vgl. Ebner 2020, S. 72) und Resilienzfaktoren werden darunter verstanden. Die Motivation setzt sich aus Teilbereichen der Identität und der psychologischen Komponenten zusammen: klare berufliche Ziele, Wichtigkeit von Arbeit respektive emotionale Verbundenheit mit der Arbeitsrolle sowie Zutrauen. Aktivitäten sind zur Gestaltung der eigenen Laufbahn wichtig. Dazu zählen proaktive Laufbahnmanagement-Verhaltensweisen wie z.B. netzwerken, 
sich über Möglichkeiten informieren, kontinuierlich lernen, planen oder sich selbst reflektieren.

\subsection{Die vier Beratungsmodule des GUIDE-Modells}

Im ersten Modul Gap wird in Anlehnung an Hirschi (2011, S. 305) eine Herausforderung in der Laufbahnentwicklung einer Person festgestellt. Anzeichen wie Angst vor einer anstehenden Reorganisation oder Hinweise aus dem Umfeld zu einem Stellenangebot können Klient/innen erkennen lassen, dass eine Veränderung ansteht (Sampson et al. 1999, S. 11). Es wird die Divergenz (der „Gap“) zwischen dem gegenwärtigen Zustand und einem erwünschten Zustand in der Zukunft erfasst. Das Anliegen wird sorgfältig analysiert. Die Beratungsperson verschafft sich ein möglichst genaues Bild zur Ausgangslage. In dieser Standortbestimmung wird die Ausprägung der Karriere-Ressourcen und der Aktivitäten zur Laufbahngestaltung ermittelt und sichtbar gemacht (BSL Oberwallis 2018, S. $51 \mathrm{f}$.). Das GUIDE-Modell wird den Klient/innen aufgezeigt, Beratungsziele und -inhalte werden schriftlich festgehalten. Eine gute, tragfähige Beratungsbeziehung wird aufgebaut.

Im zweiten Modul Understanding and Improving ,wird an den Ursachen des Anliegens gearbeitet" (Hirschi 2011, S. 308). Die Beratungsperson unterstützt die Klient/innen dabei, die Bedeutung der Ausprägung ihrer Ressourcen und Aktivitäten im Hinblick auf die Beratungsziele zu verstehen. Schwach ausgeprägte Ressourcen und Aktivitäten werden zielgerichtet entwickelt. Stark ausgeprägte Ressourcen und Aktivitäten werden im Hinblick auf die Zielsetzung genutzt. Je nach Anliegen und Ausgangslage erweitern die Klient/innen ,ihre Kenntnisse über sich selbst und über ihre beruflichen Möglichkeiten und verbinden diese beiden Bereiche“ (ebd., S. 305).

Im dritten Modul Developing solutions werden ausgehend von den gewonnenen Erkenntnissen in den vorhergehenden Modulen möglichst viele alternative Lösungen erarbeitet. Diese Handlungsmöglichkeiten werden anhand relevanter Kriterien geprüft, evaluiert und priorisiert (ebd., S. 310). Die gezielte Nutzung und Weiterentwicklung von Ressourcen und Aktivitäten werden auch in diesem Modul berücksichtigt, indem z. B. Informationen zu Arbeitsmarkt und Bildungssystem eingeholt werden.

Im vierten Modul Execution werden die Ergebnisse der Beratung in einen Handlungsplan umgesetzt. Teilziele zur Zielerreichung sowie Strategien zur Überwindung allfälliger Hindernisse werden erarbeitet. Ressourcen und Aktivitäten werden genutzt und weiterentwickelt. Es wird definiert, wann Zielfortschritte, Maßnahmen, Hindernisse und Ressourcen überprüft und gegebenenfalls angepasst werden (Hirschi 2020, S. 3). In einer Abschluss-Sitzung erfolgt die Rückkehr zum ersten Beratungsmodul. Der Beratungsprozess wird hinsichtlich der Beratungsziele reflektiert. Wenn die ursprüngliche Divergenz nicht geschlossen ist respektive die Beratungsziele nicht ausreichend erreicht sind, besteht die Möglichkeit, im zirkulären GUIDE-Modell Module erneut aufzunehmen und zu bearbeiten.

Das GUIDE-Modell lässt je nach Beratungsziel und Ausgangssituation eine umfassendere, intensivere oder eher punktuelle Begleitung der Klient/innen durch die Beratungsperson zu. Somit können die Beratungsdauer und die Anzahl der Beratungssitzungen variieren. Der Kreislauf kann in nur einer Beratungssitzung durch- 
laufen werden, wie etwa im Falle einer Entscheidungssitzung. Ein Beratungsmodul kann auch mehrere Beratungsgespräche umfassen. Ebenso besteht die Möglichkeit, zu einem vorhergehenden Modul zurückzukehren, um noch bestehende Lücken zu schließen.

\section{Das GUIDE-Modell im Beratungsalltag}

Um Klient/innen das GUIDE-Modell nachvollziehbar zu vermitteln, wurde es in der BSL Oberwallis für den Beratungstisch als Drehscheibe ${ }^{3}$ konzipiert. Bei diesem beweglichen Kompass drehen sich die Karriere-Ressourcen, angetrieben durch die Aktivitäten zur Laufbahngestaltung. Die gewonnene Zielklarheit der Klient/innen gibt letzten Endes die Kompassausrichtung vor. Mit Hilfe der Drehscheibe als Beratungsintervention können Klient/innen herausfinden, welche Aspekte für den subjektiven und den objektiven Laufbahnerfolg wichtig sind. Überdies lernen sie zentrale Prozessphasen zur Lösung von Herausforderungen in der Laufbahngestaltung kennen. Die Drehscheibe bietet somit Transparenz, Überblick, Orientierung und wirkt unterstützend beim Vereinbaren von gemeinsamen Beratungszielen, -inhalten und Prozessphasen. Die Übereinstimmung in diesen Aspekten ist, neben einer guten Bindung zwischen Klient/in und Beratungsperson, eine wesentliche Komponente für eine gute Beratungsbeziehung (Hirschi 2011, S. 307). Aus dem Einsatz der Drehscheibe ergibt sich zudem der Vorteil, dass sich eine gemeinsame Sprache zwischen Klient/in und Beratungsperson im Hinblick auf die Zielerreichung entwickeln kann (ebd., S. 303).

Jedem Beratungsmodul wurden in der BSL Oberwallis zahlreiche methodische Interventionen und Arbeitsmaterialien schwerpunktmäßig zugeordnet. Im ersten Beratungsmodul können Klient/innen z. B. anhand des Karriere-Ressourcen Fragebogens ${ }^{4}$ (CRQ; Hirschi et al. 2018) ihre Karriere-Ressourcen und Aktivitäten zur Laufbahngestaltung einschätzen. In den nachfolgenden Modulen werden die Anliegen und formulierten Beratungsziele, ausgehend von der Standortbestimmung der KarriereRessourcen, bearbeitet. So kann im zweiten Modul das Career Construction Interview (CCI; Savickas 2015) angewendet werden, um zu einem verbesserten Identitätsgefühl zu gelangen. Übungen aus der positiven Psychologie (z. B. 4-EveningQuestions; Ebner 2017) helfen, positive Emotionen zu fördern und das Wohlbefinden sowie die Motivation zu stärken. Die mentale Strategie WOOP ${ }^{5}$ (Oettingen 2015, S. 155 ff.) kann z.B. im vierten Modul die Erarbeitung und Umsetzung des Handlungsplans unterstützen und zur Förderung der Selbstmotivation dienen.

\footnotetext{
3 www.vs.ch/web/bsl/informationen-fur-fachpersonen, Zugegriffen: 15.01.2021.

4 www.cresogo.com, Zugegriffen: 15.01.2021.

5 www.woopmylife.org/de/home, Zugegriffen: 15.01.2021.
} 


\section{Fallbeispiel: Karrierecoaching nach dem GUIDE-Modell ${ }^{6}$}

Eine 45-jährige Klientin, Leiterin Human Resources, suchte in einem Karrierecoaching Unterstützung beim Planen ihrer beruflichen Zukunft. Im Erstgespräch erzählte sie von der bevorstehenden Reorganisation ihrer Abteilung, durch die sie ihre Führungsfunktion verlieren würde. Diese Veränderung belastete sie sehr. Sie führte diesen Direktionsentscheid auf einen schwelenden Konflikt zwischen ihrem Vorgesetzten und sich zurück. Ihr sei eine Mitarbeiterfunktion in dem Team, das sie aktuell führe, angeboten worden, was für sie nicht in Frage komme. Die Klientin schilderte Ideen von Laufbahnmöglichkeiten außerhalb ihrer Unternehmung, z. B. den Einstieg in die Organisation ihrer Kollegin, die ihr Personalauswahl und -entwicklung sowie Organisationsberatung anbot.

Nachdem im Erstgespräch die Ausgangslage erfasst war und die Klientin ihren Wunschzustand genauer beschrieben hatte, wurden Coaching-Ziele formuliert sowie das GUIDE-Modell anhand der Drehscheibe eingeführt. Die Klientin war sofort einverstanden, eine Analyse ihrer Karriere-Ressourcen vorzunehmen. Die Resultate des Karriere-Ressourcen-Fragebogen (CRQ) zeigten niedrige Werte bei den Ressourcen „Organisationale Unterstützung“, „Klarheit und Selbstbestimmtheit von Laufbahnzielen“. Die Ressourcen „Wichtigkeit der Arbeit“, „Entwicklungsmöglichkeiten im aktuellen Unternehmen" schätzte die Klientin hingegen eher hoch ein. Sie erklärte, dass ihre Organisation diverse interne Karriere-Perspektiven bot. Die Klientin gestand, dass sie aufgrund ihrer Enttäuschung über den Direktionsentscheid diese Ressource in letzter Zeit ausgeblendet hatte. Die Analyse ihres Bewerbungsdossiers und ihre Schilderungen ließen zudem auf viele Ressourcen in den Bereichen „Wissen und Kompetenzen“ sowie „soziale Unterstützung“ schließen, was die Resultate des Fragebogens bestätigten.

Im zweiten Gespräch war die Klientin sichtlich aufgewühlt und angespannt. Sie machte sich Sorgen über ein bevorstehendes Gespräch mit ihrem Vorgesetzten und einem Direktionsmitglied bezüglich ihrer weiteren internen Beschäftigung. Die Klientin äußerte den Wunsch, selbstbewusst und mit einer konstruktiven, aber klaren Haltung in das angekündigte Gespräch zu gehen. Sie nahm das Angebot des Coachs an, anhand einer Übung zuerst die eigene und danach die Rolle ihres Vorgesetzten einzunehmen und sich aus den verschiedenen Rollen heraus entsprechend zum Problem zu äußern. Diese Intervention ermöglichte der Klientin eine Erweiterung ihrer Perspektiven und Sichtweisen auf das Problem. Auch erarbeitete die Klientin im Gespräch mit dem Coach eine klare Vorstellung davon, welche Vorschläge sie ins bevorstehende Gespräch einbringen und worauf sie sich im Gespräch einlassen wollte. Sie erkannte, dass sie sich in diesem anspruchsvollen Gespräch auf ihre Kompetenzen verlassen konnte. Am Ende der Coaching-Sitzung zeigte sie sich wesentlich ruhiger und zuversichtlicher.

Die nächsten Sitzungen waren verschiedenen Interventionen zur Steigerung von Selbstkenntnissen (u. a. Lebenslinie; Brott 2004, S. $191 \mathrm{ff}$.) und damit verbundenen Gesprächen gewidmet. Die wichtigsten Resultate und Erkenntnisse aus diesen As-

\footnotetext{
${ }^{6}$ Im Fallbeispiel sind die Daten, die eine Identifikation ermöglichen würden, geändert, und somit ist die Anonymität gewahrt.
} 
sessments wurden zusammengefasst (Hirschi 2011, S. 309). Auch erkundigte sich die Klientin bei ihrer Kollegin über die Möglichkeit, im Bereich Personalauswahl und -gewinnung mitzuwirken. Die Klientin entwickelte Lösungsideen sowohl innerhalb als auch außerhalb ihrer aktuellen Organisation. Schließlich bewarb sie sich auf eine intern ausgeschriebene Stelle, die ihren langfristigen Zielen, Wertvorstellungen, Interessen und Stärken am ehesten entsprach. Nach einem Vorstellungsgespräch und dem Kennenlernen ihres zukünftigen Teams erhielt sie die Stelle.

Im abschließenden fünften Gespräch erarbeitete die Klientin einen Handlungsplan, der langfristige berufliche und private Ziele sowie damit verbundene kürzerfristige Ziele enthielt. Das Abschlussgespräch diente zudem der Überprüfung, ob die im ersten Beratungsmodul vereinbarten Coaching-Ziele erreicht wurden. Veränderungen in den Karriere-Ressourcen wurden durch den erneuten Einsatz des KarriereRessourcen Fragebogens gemessen und besprochen. Der Coaching-Prozess wurde reflektiert.

\section{Erkenntnisse aus der Arbeit mit dem GUIDE-Modell}

Das Fallbeispiel verdeutlicht, dass der Beratungsprozess nach dem GUIDE-Modell nicht zwingend linear verlaufen muss. In der zweiten Sitzung wurden nebst der Entwicklung von psychologischen Ressourcen und Identitätsressourcen bereits mögliche Lösungen angedacht. In den darauffolgenden Sitzungen erweiterte die Klientin verstärkt ihre (Selbst)kenntnisse, um schließlich weitere Lösungen zu generieren. Anhand der Drehscheibe hat die Klientin in den Folgegesprächen ihre Fortschritte in der Laufbahnplanung überprüft und gemeinsam mit dem Coach Sitzungsziele vereinbart. Die Visualisierung des Modells hat dazu beigetragen, dass die Klientin ihre Laufbahnentwicklung besser verstehen konnte und dass sie sich gut auf den Beratungsprozess eingelassen hat, was eine wichtige Grundlage für eine Verbesserung der Handlungssteuerung ist (Loebbert 2018, S. 25). Die Klientin verfügte über ideale Voraussetzungen (Anliegen, persönliche Eigenschaften), um von Coaching respektive Prozessberatung zu profitieren. Nach Ebner (2016, S. 84) helfen die beiden Eigenschaften Selbstreflexion und Proaktivität, zu erklären, wie Klient/innen von einem Coaching profitieren, wobei Proaktivität laut Ebner definiert ist als ,die Motivation einer Person, eine gegebene Situation zu verändern und dabei sowohl Hindernisse wie auch Probleme zu antizipieren und zu bewältigen“.

Wie kann in der Beratung nach dem GUIDE-Modell im Individualfall abgeschätzt werden, wie viel Prozessberatung und wie viel Expertenberatung für eine nachhaltig wirksame Beratung notwendig sind? Eine mögliche Antwort könnte darin bestehen, dass die Beratungsperson im ersten Beratungsmodul in der Prozessberatungsrolle startet (vgl. Schein 2017, S. 38). Die Beratungsperson konzentriert sich in Anlehnung an Hirschi (2011, S. 307) auf den Aufbau einer tragfähigen Beratungsbeziehung und versucht, das Anliegen, die Ausgangslage und vorhandene Ressourcen der Klient/ innen genau zu erfassen. Davon ausgehend schätzt sie ab, welche Interventionen sich am besten eignen respektive wie viel Wissen vermittelt werden muss, um die Klient/ innen erfolgreich in ihrer Handlungssteuerung zu unterstützen. Die Beratungsperson tauscht sich mit den Klient/innen über das gemeinsame Rollenverständnis im 
Beratungsprozess aus und bewegt sich auch im weiteren Beratungsverlauf bewusst auf dem Kontinuum zwischen Prozessberatung und Expertenberatung (vgl. Loebbert 2018, S. 11).

Um Klient/innen wirksam in der Gestaltung ihrer Laufbahn zu unterstützen, benötigen Beratungspersonen vielfältige Kompetenzen (vgl. Handbuch NICE Network ${ }^{7}$ sowie Hirschi 2019, S. 750). Beratungspersonen sind gefordert, über aktuelles Wissen in unterschiedlichen Themenfeldern (Prozesskenntnisse, Wissen zur Prozesssteuerung, psychologisches Wissen, Wissen zum Arbeitsmarkt usw.) zu verfügen. Um dieses Wissen im Beratungsprozess wirksam und zielführend zum Tragen zu bringen, ist Rollenbewusstsein, Professionalität und reflektierte Erfahrung gefragt. Das Guide-Modell, das in jeder Beratungssequenz allen Beteiligten als Kompass offen vorliegt, sorgt für inhaltliche Orientierung und einen zielgerichteten Beratungsablauf.

Open Access Dieser Artikel wird unter der Creative Commons Namensnennung 4.0 International Lizenz veröffentlicht, welche die Nutzung, Vervielfältigung, Bearbeitung, Verbreitung und Wiedergabe in jeglichem Medium und Format erlaubt, sofern Sie den/die ursprünglichen Autor(en) und die Quelle ordnungsgemäß nennen, einen Link zur Creative Commons Lizenz beifügen und angeben, ob Änderungen vorgenommen wurden.

Die in diesem Artikel enthaltenen Bilder und sonstiges Drittmaterial unterliegen ebenfalls der genannten Creative Commons Lizenz, sofern sich aus der Abbildungslegende nichts anderes ergibt. Sofern das betreffende Material nicht unter der genannten Creative Commons Lizenz steht und die betreffende Handlung nicht nach gesetzlichen Vorschriften erlaubt ist, ist für die oben aufgeführten Weiterverwendungen des Materials die Einwilligung des jeweiligen Rechteinhabers einzuholen.

Weitere Details zur Lizenz entnehmen Sie bitte der Lizenzinformation auf http://creativecommons.org/ licenses/by/4.0/deed.de.

\section{Literatur}

Andres Roduit, C., Walter, M.-C., Le Stanc, F., \& Zurbriggen, E. (2018). Ein Kompass für den Beratungsprozess. Panorama, 2, 24-25.

Brott, P.E. (2004). Constructivist assessment in career counseling. Journal of Career Development, 30(3), 189-200.

BSL Oberwallis (2018). Beratungskonzept. http://www.vs.ch/web/bsl/beratungskonzept. Zugegriffen: 15. Jan. 2021

BSL Oberwallis (2020). Beschreibung GUIDE-Modell. http://www.vs.ch/web/bsl/beratungskonzept. Zugegriffen: 8. Feb. 2021.

Ebner, K. (2016). Veränderungen durch Coaching: Wie lernt ein Coachee im Coaching-Prozess? In C. Triebel, J. Heller, B. Hauser \& A. Koch (Hrsg.), Qualität im Coaching (S. 81-91). Berlin, Heidelberg: Springer.

Ebner, K. (2020). Karriereoptimismus im Karrierecoaching fördern und messen. Organisationsberatung, Supervision, Coaching, 27(1), 65-78.

Ebner, M. (2017). 4-Evening-Questions: Eine einfache Technik mit tiefgreifender Wirkung. Organisationsberatung, Supervision, Coaching, 24(3), 269-282.

Ebner, K., \& Kauffeld, S. (2019). Laufbahngestaltung durch Karrierecoaching. In S. Kauffeld \& D. Spurk (Hrsg.), Handbuch Karriere und Laufbahnmanagement (S. 759-784). Berlin: Springer.

Greif, S. (2008). Coaching und ergebnisorientierte Selbstreflexion. Göttingen: Hogrefe.

Gubler, M. (2019). Neue Laufbahnmodelle in Theorie und Praxis: Eine kritische Würdigung. In S. Kauffeld \& D. Spurk (Hrsg.), Handbuch Karriere und Laufbahnmanagement (S. 937-962). Berlin: Springer.

\footnotetext{
7 www.nice-network.eu/pub/, Zugegriffen: 15.01.2021.
} 
Gulder, A. (2013). Finde den Job, der dich glücklich macht (3. Aufl.). Frankfurt/New York: Campus.

Haenggli, M., Marciniak, J., \& Hirschi, A. (2019). Die Rolle von Karriere-Ressourcen für eine lebenslange Laufbahngestaltung. Zeitschrift des Deutschen Verbandes für Bildungs- und Berufsberatung e. V., 1 , 4-9.

Hall, D. T. (2004). The protean career: a quarter-century journey. Journal of Vocational Behavior, 65(1), 1-13.

Hirschi, A. (2011). Wirksames Karriere-Coaching: Ein Grundlagenmodell. Organisationsberatung, Supervision, Coaching, 18(3), 301-315.

Hirschi, A. (2012). The career resources model: an integrative framework for career counsellors. British Journal of Guidance \& Counselling, 40(4), 369-383.

Hirschi, A. (2015). Konzepte zur Förderung von Laufbahnentwicklung im 21. Jahrhundert. In R. Zihlmann \& D. Jungo (Hrsg.), Berufswahl in Theorie und Praxis (4. Aufl. S. 65-82). Bern: SDBB.

Hirschi, A. (2019). Berufs- und Laufbahnberatung. In S. Kauffeld \& D. Spurk (Hrsg.), Handbuch Karriere und Laufbahnmanagement (S. 739-758). Berlin: Springer.

Hirschi, A. (2020). Whole-life career management: a counseling intervention framework. Career Development Quarterly, 68, 2-17.

Hirschi, A., Haenggli, M., Nagy, N., Baumeler, F., Johnston, C., \& Spurk, D. (2019). Karriere-Ressourcen messen: Validierung der deutschsprachigen Version des Karriere-Ressourcen Fragebogens. Diagnostica, 65(3), 133-141.

Hirschi, A., Nagy, N., Baumeler, F., Johnston, C.S., \& Spurk, D. (2018). Assessing key predictors of career success: development and validation of the career resources questionnaire. Journal of Career Assessment, 26(2), 338-358.

Hirschi, A. (2018). Berufs-, Studien- und Laufbahnberatung. www.sbfi.admin.ch/sbfi/de/home/dienstleis tungen/publikationen/publikationsdatenbank/bericht-bslb.html. Zugegriffen: 9. Febr. 2021. Bericht im Auftrag des Staatssekretariats für Bildung, Forschung und Innovation SBF im Rahmen des Projekts „Berufsbildung 2030 - Vision und Strategische Leitlinien“.

Kauffeld, S., Güntner, A. V., \& Ebner, K. (2018). Karriere als Thema im Coaching. In S. Greif, H. Möller \& W. Scholl (Hrsg.), Handbuch Schüsselkonzepte im Coaching (S. 285-294). Berlin: Springer.

Lang-von Wins, T., \& Triebel, C. (2012). Karriereberatung (2. Aufl.). Berlin, Heidelberg: Springer.

Latzke, M., Schneidhofer, T. M., Mayrhofer, W., \& Pernkopf, K. (2019). Karriereforschung: Konzeptioneller Rahmen, zentrale Diskurse und neue Forschungsfelder. In S. Kauffeld \& D. Spurk (Hrsg.), Handbuch Karriere und Laufbahnmanagement (S. 3-35). Berlin: Springer.

Loebbert, M. (2018). Coaching in der Beratung. Wiesbaden: Springer.

Masdonati, J., Massoudi, K., \& Rossier, J. (2019). Repères pour l'orientation. Lausanne: Antipodes.

Oettingen, G. (2015). Die Psychologie des Gelingens. München: Pattloch.

Rappe-Giesecke, K. (2008). Triadische Karriere-Beratung. Bergisch Gladbach: EHP.

Rauen, C. (2014). Coaching (3. Aufl.). Göttingen: Hogrefe.

Sampson, J.P., Lenz, J. G., Reardon, R.C., \& Peterson, G. W. (1999). A cognitive information processing approach to employment problem solving and decision making. The Career Development Quarterly, 48(1), 3-18.

Savickas, M.L. (2015). Life-design counseling manual. www.vocopher.com. Zugegriffen: 15. Jan. 2021.

Scheidt, B. (2009). Neue Wege im Berufsleben (3. Aufl.). Offenbach: GABAL.

Schein, E. H. (1975). How career anchors hold executives to their career paths. Personnel, 52(3), 11-24.

Schein, E. H. (1978). Career dynamics: matching individual and organizational needs. Reading, MA: Addison-Wesley.

Schein, E.H. (2004). Karriereanker. Die verborgenen Muster in Ihrer beruflichen Entwicklung (9. Aufl.). Darmstadt: Lanzenberger Dr. Looss Stadelmann.

Schein, E.H. (2010). Prozessberatung für die Organisation der Zukunft (3. Aufl.). Bergisch Gladbach: EHP.

Schreiber, M. (2020). Wegweiser im Lebenslauf. Stuttgart: Kohlhammer. 


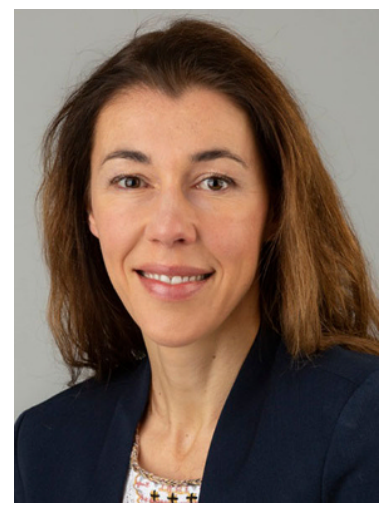

Christine Andres Roduit MSc in Arbeits- und Organisationspsychologie, Dipl. Berufs-, Studien- und Laufbahnberaterin. Sie verfügt über langjährige Erfahrung in der Personalgewinnung und -entwicklung verschiedener Unternehmen. Seit fast 10 Jahren ist sie als Berufs- und Laufbahnberaterin sowie als Karrierecoach tätig.

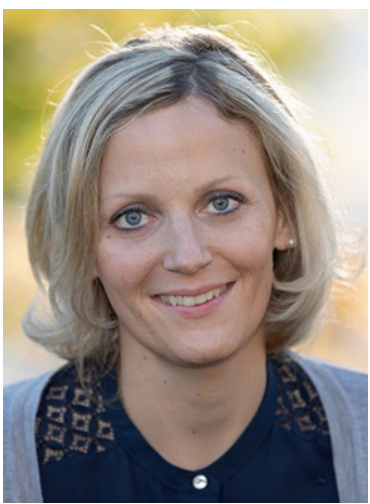

Marie-Christine Walter MSc in Pädagogik und Pädagogische Psychologie, Dipl. Berufs-, Studien- und Laufbahnberaterin, verfügt über Arbeitserfahrung im HR- und Schulbereich und ist seit 2009 als Berufsund Laufbahnberaterin sowie als Karrierecoach tätig. 